Original Research

\title{
Variations in Secondary Health Resource Utilization Post Clozapine Initiation
}

Prasanna N. de Silva *

Cumbria, Northumberland, Tyne and Wear NHS Foundation Trust, Monkwearmouth Hospital, Newcastle Road, Sunderland SR1, UK; E-Mail: Prasanna.desilva@cntw.nhs.uk

* Correspondence: Prasanna N. de Silva; E-Mail: Prasanna.desilva@cntw.nhs.uk

Academic Editor: Bart Ellenbroek

Special Issue: $\underline{\text { Recent Advances in Psychiatry }}$

OBM Neurobiology

2020, volume 4, issue 4

doi:10.21926/obm.neurobiol.2004078
Received: September 09, 2020

Accepted: November 17, 2020

Published: November 26, 2020

\begin{abstract}
This study looked at secondary care utilization metrics as an effectiveness indicator of Clozapine initiation in 77 patients over the following year compared to the year prior to initiation. It was found that there was a marginal drop in psychiatric bed utilization, alongside reduced crisis team use. Medical and psychiatric outpatient use increased, as did physical health interventions. Overall, compared with costs over the year preceding Clozapine initiation, an estimated saving per patient of $£ 3,867(\$ 5,065)$ was achieved.
\end{abstract}

\section{Keywords}

Psychosis; Clozapine; health economics

\section{Introduction}

Clozapine, classified as an 'atypical' antipsychotic, has a range of actions on dopaminergic, serotoninergic, histaminergic, cholinergic and adrenergic receptors [1]. This balanced activity profile

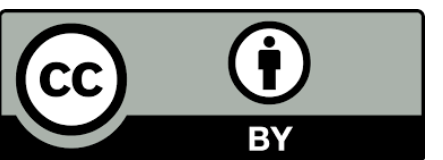

(C) 2020 by the author. This is an open access article distributed under the conditions of the Creative Commons by Attribution License, which permits unrestricted use, distribution, and reproduction in any medium or format, provided the original work is correctly cited. 
appears to confer this drug freedom from extrapyramidal side effects, alongside effectiveness in negative symptoms of psychosis (such as apathy), and improvements in cognitive deficits (attention, recall, executive functioning). There is also evidence of reduced suicidality post Clozapine initiation [2]. Consequently, Clozapine remains the treatment of choice for resistant psychosis [3] with evidence of improvement around 55\% [4]. Predictors of good outcome has been described previously [5] (Lieberman et.al, 1994), alongside health economic outcome data in North America involving patients with Schizophrenia [6, 7].

However, a National Health Service (NHS) Trust wide study of objective metrics such as hospital bed use, psychiatric / medical outpatient episodes, physical health monitoring and crisis team contacts have not been carried out. It is also unknown if specific sub groups, such as patients with premorbid criminal and substance use behaviour have outcomes similar to those without such comorbidities. Furthermore, correlation between objective metrics and clinician opinions on effectiveness has not been analysed.

\section{Objectives of Study}

1. To confirm the overall effectiveness figure (as a \%) as documented by the treating psychiatrist at 1 year post clozapine initiation, to compare this with figures provided by previous studies.

2. To compare bed days, psychiatric outpatient use, medical outpatient contacts, crisis team contacts, and physical health contacts during the 12 months preceding Clozapine initiations versus 12 months post initiation, and to use hypothecated costing to describe the effect size of secondary healthcare costs.

3. To ascertain the extent of additional psychotropic use (such as other atypical antipsychotics, benzodiazepines, Mood Stabilisers and Antidepressants) to augment Clozapine at the end of 1 year.

\section{Inclusion and Exclusion Criteria}

1. Adults with non-organic psychoses (schizophrenia, schizoaffective and delusional disorders) between the ages of 18-65 (both sexes) included. Those with organic psychosis related to traumatic brain Injury, epilepsy and Parkinson's disease excluded.

2. Note taken of behaviours existing prior to psychosis diagnosis involving criminal conduct and substance abuse features; as these behaviours have been clinically known to influence outcomes.

3. Clozapine initiated between 2010 and 2018 (data integrity assured on Rio electronic database).

\section{Hypotheses}

Clozapine initiation will reduce hospital bed, out patient and crisis team utilisation over the next year, with equivalent reductions in cost of healthcare for secondary mental health services.

\section{Method.}

Ethics approval was granted by the R\&D department. Furthermore, approval was sought from the Trust Caldecott guardian to access patient records. Thereafter the author accessed Trust pharmacy services for the list of patients attending the 3 Clozapine clinics within the Trust (North, Central and South business units). 
The author systematically reviewed the documentation letters, identifying features of initial presentation, diagnoses (including comorbidities), date of initiation on Clozapine and ascertained bed use (in terms of the number of months corrected down), outpatient, crisis team and physical healthcare contacts. Evidence of other psychotropics alongside Clozapine was noted at the end of the 1 year period on Clozapine. Clinician observed outcome was also noted at the end of 1 year on Clozapine by examining the most recent letter to primary care at 1 year following Clozapine initiation.

Hypothecated costs were estimated as $£ 12,000$ bed cost per month (a month calculated as 30 days), psychiatric outpatient appointment cost $f 300$ each, medical outpatient appointment cost $£ 400$, crisis team episode cost $£ 200$ and physical healthcare episode $f 100$. It was agreed that the annual cost of Clozapine prescriptions was $£ 500$ per patient, taking in to account the use of liquid Clozapine used in some ward settings on initiation, which incurs a higher cost.

\section{Results}

\subsection{Subject Characteristics}

Overall, 77 subjects were included in the study; 44 males and 33 females between the ages of 23 and 65 years. Regards main diagnosis, the vast majority; 59 (76\%) were diagnosed to have Schizophrenia (including 3 people with coexisting Learning Disability), 9 with Schizoaffective disorder, 4 with Delusional Disorder and 5 with Bipolar Affective Disorder. Of the 77 patients, prior to Clozapine initiation, 15 (19\%) were noted to be abusing substances (alcohol, cannabis, amphetamine) and 11 (14\%) patients had criminal convictions of which 5 were under the care of forensic psychiatry.

\subsection{Clinicians Judgements}

According to their treating consultant psychiatrist, $43 / 77$ (55\%) responded to Clozapine at the end of 1 year post initiation, as evidenced by clinic letters. Partial or non-responders were characterised by social avoidance, continuing substance use (including alcohol) and continuing hallucinations. Supported housing and family involvement was associated with clinician judgements of good response. Regards the 11 patients with premorbid criminal convictions (including 5 treated in a forensic setting) the treating psychiatrists were largely satisfied by their clinical improvement; for example reduced challenging behaviour and absconsion from wards.

\section{Healthcare Utilisation}

Regards service utilisation, there was a marginal (4\%) reduction of bed use from a total of 274 to 262 months. Crisis team utilisation fell from 37 to 12 episodes (a 67\% reduction). Psychiatric outpatient use marginally increased; 200 appointments offered before Clozapine initiation versus 216 after. There was a rise of medical outpatient contacts; 47 to 78 ; a $40 \%$ increase, mainly involving cardiac and diabetic clinic attendances. There was also a rise in physical health care appointments; 52 to 100 (a 50\% increase), mainly for exercise sessions. Patients with premorbid criminal convictions did not show increased bed use post Clozapine initiation, but those abusing substances and alcohol did (a rise from 78 to 94 months in total). 


\section{Hypothecated Financial Costs and Savings}

On overall costs, overall Clozapine initiation produced a cost saving of $f 297,800$ for the 77 patients in the first year, a hypothecated saving of $£ 3867$ per patient. On bed use there was a cost reduction of $£ 348,000$. Crisis team use showed a reduction of $£ 5,000$ alongside cost increases on psychiatric outpatient use $£ 4,800$. There were increases in medical out-patient costs by $£ 12,400$ and physical healthcare by $f 4,800$. The overall cost of Clozapine prescription (including level testing) was estimated to be $£ 38,500$ (in excess of pre Clozapine atypical antipsychotic use).

\section{Discussion}

This is the first published study examining overall health utilisation metrics (bed days, outpatient and crisis team episodes) over the first year following Clozapine initiation involving the full range of diagnostic groups (including people with comorbid learning disability). There are other studies modelling costs of Clozapine use; mainly North American data specifically studying patients with a diagnosis of schizophrenia [6] and Bipolar disorder [8]. These studies showed cost savings in terms of reduced bed use 2 years post clozapine initiation. However, community health costs and pharmacy costs were not described.

Regards the hypotheses, this study showed that Clozapine initiation did not significantly reduce hospital bed use or outpatient contacts, but crisis team use was reduced over the next year, with equivalent reductions in cost, albeit marginal. However, this study does suggest that cost savings are evident even within the first year. There was evidence that community patients continuing to misuse substances showed more bed usage; perhaps the substance abuse was a symptom of untreated psychosis needing additional anti-dopaminergic medication, or treatments specifically targeting the behaviour (such as motivational interviewing or Methadone replacement). Prior to Clozapine initiation, all the subjects had received between 4 and 5 other antipsychotic medication serially (without evidence of antipsychotic polypharmacy), in addition to benzodiazepine anxiolytics and serotonergic antidepressants. Following Clozapine initiation, there was evidence of reductions in benzodiazepines and antidepressants.

There were 10 patients who continued to receive other atypical antipsychotics at 1 year, mainly atypical anti-dopaminergic drugs. Patients on additional atypical antipsychotics showed clinical improvement according to their treating psychiatrists, suggesting potential benefit of augmenting Clozapine with selective Dopamine blockers such as Amisulpiride (as was the case in this study). This has been commented on in other studies [9]. This sample was too small to detect any clear reduction of self-harm attempts as only 6 patients presenting with suicidality prior to Clozapine use. Certainly there was no evidence of increased suicidality post Clozapine initiation. Interrogation of multiple electronic databases of NHS mental health trusts could answer this important question, including looking at inflammatory markers as a predictor of suicidality [10].

On weaknesses, this was a retrospective study covering 10 years of Clozapine initiation; the consequence of the need to maximise the number of patients used for analysis. Clinical practice would have changed during this period regards selection for Clozapine initiation, for example including those with delusional disorder and bipolar affective disorder. The financial estimates pertain to a single NHS Trust, and might not be representative.

\section{Service Developments}


In order to reduce drug costs, ward staff have been asked to discontinue the use of liquid Clozapine (6 times the cost of oral Clozapine). Liquid Clozapine appears to have been used as means of achieving concordance; but directly observed ingestion as part of an interview would be equally effective. Assertive management of substance misuse (including the use motivational interviewing and Methadone replacement), is also being encouraged, both to reduce costs and improve clinical outcomes. A case has been made for mobile Clozapine testing to reduce bed use simply awaiting haematology and Clozapine level testing results.

The Trust is currently upgrading its electronic database to produce mandatory alerts if a patient is on Clozapine, Lithium and depot antipsychotics. This would significantly improve safety in terms of potential drug interactions and toxicity. Improvements in pre initiation consent practice including achieving better patient awareness of metabolic risks - has been recommended, including improved documentation of discussions.

\section{Conclusions}

This study showed clinical improvement rates similar to previous studies on Clozapine initiation; around $55 \%$ of initiators showing improvement at 1 year. Clinical impressions appear to be backed up by reduced health utilisation metrics, especially regards crisis team involvement. Use of liquid Clozapine in ward settings, for maintaining concordance is to be avoided to reduce costs further, alongside more flexible community blood and level checks. Furthermore, additional interventions to manage substance misuse among Clozapine users is necessary to improve outcome, alongside supportive infrastructure including housing. An additional feature worthy of note is the method employed in this study to assess Clozapine cost efficacy; which could be used to assess cost utilisation for other drugs such as mood stabilisers, stimulants and memory enhancers.

\section{Author Contributions}

Dr Prasanna N. de Silva is the sole author, responsible for study design, data collection, presentation of findings and drafting manuscript.

\section{Competing Interests}

The author has declared that no competing interests exist.

\section{References}

1. Stahl SM. The prescriber's guide: Stahl's essential psychopharmacology. 6th ed. New York: Cambridge University Press; 2017.

2. Taipale H, Lahteenvuo M, Tanskanen A, Mittendorfer-Rutz E, Tiihonen J. Comparative effectiveness of antipsychotics for risk of attempted or completed suicide among persons with schizophrenia. Schizophr Bull. 2020: sbaa111.

3. Kane JM, Marder SR, Schooler NR, Wirshing WC, Umbricht D, Baker RW, et.al. Clozapine and Haloperidol in moderately refractory schizophrenia; a 6 month randomised and double blind comparison. Arch Gen Psych. 2001; 58: 965-972.

4. Raguraman J, Sagar KJ, Chandrasekaran R. Effectiveness of Clozapine in treatment resistant schizophrenia. Indian J Psychiatry. 2005; 47: 102-105. 
5. Lieberman JA, Safferman SA, Pollack S, Szymanski S, Johns C, Howard A, et al. Clinical effects of Clozapine in chronic schizophrenia: Response to treatment and predictors of outcome. Am J Psychiatry. 1994; 151: 1744-1752.

6. Meltzer HT, Cola P, Way L, Thompson PA, Bastani B, Davies MA, et.al. Cost effectiveness of Clozapine in neuroleptic resistant schizophrenia. Am J Psychiatry. 1993; 150: 1630-1638.

7. Rosenheck R, Cramer J, Allan E, Erdos J, Frisman LK, Xu W, et al. Cost effectiveness of Clozapine in patients with high and low levels of hospital use. Arch Gen Psychiatry. 1999; 56: 565-572.

8. Nielsen J, Kane JM, Cornell C. Real world effectiveness of Clozapine in patients with Bipolar disorder: Results of a mirror image study. Bipolar Disorders. 2012; 14: 863-869.

9. Porcelli, S., Balzarro, B., Serretti, A. Clozapine resistance: Augmentation strategies. Eur Neuropsychopharmacol. 2012; 22: 165-182.

10. Brundin L, Erhardt S, Bryleva EY, Achtyes ED, Postolacheet TT. The role of inflammation in suicidal behaviour. Acta Psychiatr Scand. 2015; 132: 192-203.

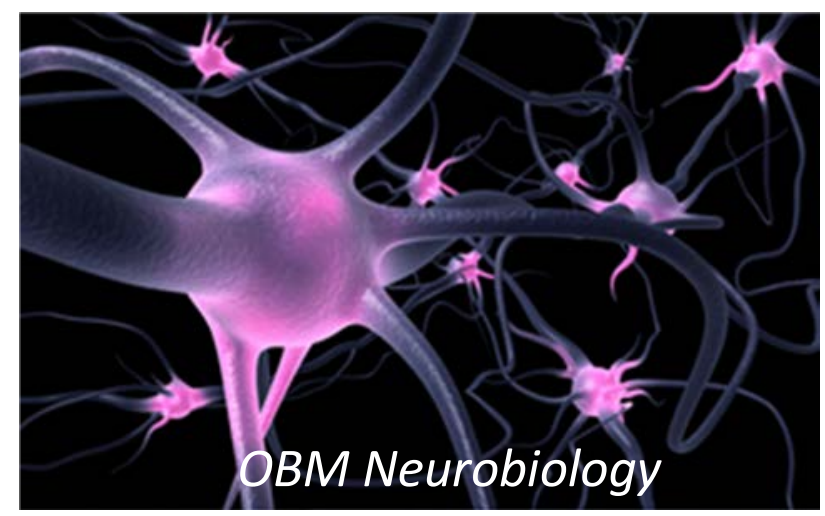

Enjoy OBM Neurobiology by:

1. Submitting a manuscript

2. Joining volunteer reviewer bank

3. Joining Editorial Board

4. Guest editing a special issue

For more details, please visit:

http://www.lidsen.com/journals/neurobiology 